\title{
Latest advances of bioprinting in space: an interview with Michael Gelinsky
}

\author{
Michael Gelinsky*,1 \\ ${ }^{1}$ Head, Centre for Translational Bone, Joint \& Soft Tissue Research, University Hospital \& Faculty of Medicine, TU Dresden (Dresden \\ University of Technology), Fetscherstr. 74, 01307 Dresden, Germany \\ *Author for correspondence: michael.gelinsky@tu-dresden.de
}

"We propose that plasma could be obtained from the astronaut for whom the tissue construct is being printed - and biopolymers (such as alginate, cellulose, gellan gum etc.) can be isolated from algae, plants or bacteria, which can be cultivated in the spaceship or the future extra-terrestrial settlements."

First draft submitted: 6 December 2019; Accepted for publication: 28 January 2020; Published online: 10 February 2020

Keywords: additive manufacturing • astronaut • biofabrication • bioprinting • extra-terrestrial settlements $\bullet$ space exploration • Space flight

Michael Gelinsky received his PhD in Chemistry from Freiburg University (Germany). In 1999, he moved to Dresden University of Technology (Germany) and worked for around 10 years in the Department of Materials Science, heading his own group at the newly founded Max Bergmann Center of Biomaterials from 2002. In 2010, he was appointed Professor at the Faculty of Medicine and is currently head of the Centre for Translational Bone, Joint and Soft Tissue Research. Gelinsky's work is focused on biomaterials and scaffold development, tissue engineering and regenerative therapies, mostly for musculoskeletal tissues. His group is also very active in the field of additive manufacturing (AM) of implants and biofabrication technologies.

\section{Interview questions}

How were you initially introduced to 3D printing technologies? What made you decide to adopt 3D printing \& bioprinting into your work in bioengineering?

My lab has been working on different methods for scaffold fabrication, with a special emphasis on achieving anisotropic structures, since the tissues of our bodies also have distinct anisotropic morphologies. Our group started to work with AM approximately 10 years ago and decided to start with extrusion printing. In the beginning, we used this technology only for fabrication of biomaterial scaffolds but later, we moved into bioprinting living cells. For this, we developed our own bioinks, consisting of biopolymer blends and composites, and then utilized these inks for several cell types, including nonmammalian cells like microalgae and plant-derived cells, for which we have coined the term 'green bioprinting'. In the meantime, we are also using fused filament fabrication, melt electrowriting and inkjet-based AM technologies and study various combinations of these methods for different applications.

\section{Could you describe your recent work studying bioprinting in space? How did you become involved} with the area of space research?

In 2017, the European Space Agency (ESA) published a call for proposals to investigate '3D printing of living tissues for space exploration' and OHB System AG (Bremen, Germany) asked our group whether we would be interested in working together on this study. Our proposal, led by Sandra Podhajsky and Klaus Slenzka (both OHB System AG), was selected by the ESA, so we started to investigate how bioprinting could be used in future exploratory space missions and maybe, for human settlements on the Moon or Mars, which are visions the space agencies have started to work on concretely. My lab had no prior experience in space-related research topics, making this an exciting new subject area for us. However, as OHB System AG is the biggest German satellite company, together, 
we have expertise in the aspects of both space flight and bioprinting. Since the start of the project in May 2018, we have had several meetings with the head of the study, Tommaso Ghidini, at the European Space Research and Technology Centre (Noordwijk, The Netherlands), and have also organized an expert workshop on bioprinting in space at the center.

\section{Why is it important to have bioprinting in space? What are the potential applications for this research?}

Human exploratory missions to the Moon or Mars have long been widely considered as the next logical steps in human space exploration and, more recently, settlement. Such activities involve long-term exposure of humans to space conditions and in the case of increasing distances to Earth, no possibility of returning for medical treatment. To protect human lives and health, such space exploration missions therefore have to consider a medical infrastructure on board a spacecraft and ultimately on the Moon or Mars, so that medical treatment of a wide range of health issues can be provided. It is important to also keep in mind that only a few astronauts can participate in such missions, meaning any medical infrastructure needs to operate at least in a semi-automated manner. Bioprinting shows promise to offer treatment options for accidental injuries that are likely to happen during long-term space exploratory missions and extra-terrestrial human settlements; for example, the therapy of severe burns or difficult bone fractures.

\section{What about other applications of AM technologies in space, aside from bioprinting?}

$\mathrm{AM}$ is probably most developed in the aerospace field and major aviation companies already use AM for the production of parts, mostly for weight reduction purposes. In the case of long-term manned space missions and extra-terrestrial human settlements, AM will play a very important role based on the potential use of in situ resources and the high recycling potential, since the load which can be carried to space is extremely limited. This means that it is impossible to carry, for example, all possible spare parts for the spacecraft and machines to be used on the Moon or Mars. It will therefore be much more effective to bring a 3D printer and suitable raw materials so that the necessary parts can be produced on demand. The same principle applies to medical treatments. For example, very specific surgical instruments that might be needed for certain procedures cannot be brought from Earth, so should be produced on-site. The ESA is already investigating robots equipped with 3D printers which can autonomously build houses on the Moon using sunlight and lunar material. It has actually been suggested that those could be transported to the Moon first, so that astronauts arriving there for long-term stays will find ready-built houses on arrival. In my opinion, spaceflight is a strong driving force for current AM technology and material development.

\section{How does reduced gravity affect bioprinting? Have you been able to test these effects?}

Together with our colleagues from OHB System AG, we have looked into this. First, bioprinting includes liquids, such as cell culture media and suspended live cells, which are not easy to handle in space, especially under reduced gravity conditions. However, for such technical problems, solutions have already been found, as demonstrated by the numerous long-running cell culture experiments being carried out in space, such as at the International Space Station.

Concerning gravity, however, we must keep in mind that the absence of gravity is only present during space flight, whereas on Moon and Mars, gravity does apply, albeit reduced compared with that on Earth. When you consider the medical application of bioprinted tissue constructs, surgical interventions may apply which, again, are difficult to perform at zero gravity but research is also progressing in this area.

Within this short-term project, we were unable to send any samples to space or to use the typical model experiments like parabolic flights or drop tower studies, in which zero gravity can be simulated for short-time periods. In the early stages of our research, however, we tested micro-extrusion printing upside down, which means the $\mathrm{g}$-force was turned to 180 degrees compared with normal. This surprisingly worked quite well and we were able to print well-defined constructs consisting of more than 50 layers, both with a typical biopolymer hydrogel bioink and with a pasty calcium phosphate bone cement, a material we are using for mechanical support in bone bioprinting. We therefore predict that the bioprinting process in space should not be too problematic, even at zero gravity, provided the materials possess sufficient adhesiveness. 
What are some other challenges related to bioprinting in space \& how do you propose we could address these in the future?

As I mentioned, perhaps the most critical limitation and significant difference compared with the situation on Earth is the highly restricted loading capacity and limited available materials. For example, if you keep in mind how much cell culture media one needs just to achieve suitable cell numbers and how much single-use plasticware is normally used, this would not be possible in space. We therefore have to consider renewable sources for most materials needed and guarantee that all the materials used can be recycled.

We have actually developed a bioink that is based on human plasma and biopolymers, and therefore solely from renewable sources. We propose that plasma could be obtained from the astronaut for whom the tissue construct is being printed - and biopolymers (such as alginate, cellulose, gellan gum etc.) can be isolated from algae, plants or bacteria, which can be cultivated in the spaceship or the future extra-terrestrial settlements. We are therefore confident that bioprinting can work in space despite the difficult boundaries.

\section{Where do you think the field of bioprinting in space will be in the next 5-10 years?}

The field of bioprinting in general will continue to advance and I am convinced that we will soon see more applications, even though the field is still focused on miniaturized human tissue models. We hope to gain further funding to continue our own research into bioprinting in space. I am quite sure that the space agencies worldwide will recognize bioprinting as a highly interesting tool for fabrication of more complex $3 \mathrm{D}$ tissue models for research. For example, it would be interesting to use these tissue models to study the impact of radiation, as well as how microgravity influences cell behavior and intercellular interactions. The use of such bioprinted 3D tissue models is much closer to the animal or human body, compared with conventional cell cultures. We will therefore see bioprinted tissues being sent to space and bioprinters being installed at the International Space Station or other spacecrafts, so that such tissue-like constructs can be fabricated in space.

\section{What are some of the challenges facing the general field of bioprinting \& its application?}

If we want to apply bioprinted tissue constructs for medical treatments of humans, either on Earth or in space, we need to integrate a functional vascular supply which can be microsurgically connected to the arteriovenous system of the patient. This is the main limitation for the clinical application of bioprinting, or even tissue engineering in general, with the exception of few nonvascularized tissues like articular cartilage or thin skin samples. In addition, it would be helpful not only for the utilization of bioprinting in space but also for the possible approval of bioprinted constructs for clinical applications if we could use standardized biomaterials (i.e., bioinks), which only need to be slightly modified for different tissues or cell types.

Finally, it has become clear to me that there is not one bioprinting technology that covers all aspects needed to build functional, tissue-like constructs in clinically relevant dimensions. We should therefore look more into combinations of different technologies such as extrusion and inkjet-based bioprinting, and also investigate further multimaterial printing as it will be difficult to print sufficiently robust tissues from cell-loaded bioinks only.

\section{Is there any other exciting research in the field we should be looking out for?}

$\mathrm{AM}$, and especially $3 \mathrm{D}$ bioprinting, is developing very quickly and we should therefore see tremendous progress concerning novel technologies, materials and strategies for printing of functional tissue constructs. Many of these developments are also relevant for application in space and therefore should be reviewed thoroughly.

Of course, we also have to study what is happening in space research. For example, recently, the Russian space agency, Roscosmos, tested a device called 'Organ.Aut' for assembly of cell aggregates using magnetic fields (which could be classified as a novel bioassembly technology) in space; a method that works even better in the absence of gravity compared with the conditions on Earth. However, as this method requires the use of toxic chemicals, future medical applications are questionable.

\section{Financial and competing interests disclosure}

M Gelinsky's lab has carried out research on the topic of the interview within a study, funded by the European Space Agency through its General Study Program scheme. In the study, his lab acted as subcontractor of OHB Systems AG, Bremen, Germany. The author has no other relevant affiliations or financial involvement with any organization or entity with a financial interest in or financial conflict with the subject matter or materials discussed in the manuscript apart from those disclosed.

No writing assistance was utilized in the production of this manuscript. 
Interview Gelinsky

Interview disclosure

The opinions expressed in this interview are those of M. Gelinsky and do not necessarily reflect the views of Future Medicine Ltd/Future Science Ltd/Newlands Press Ltd. 